\title{
Near-Capacity Turbo Coded Soft-decision Aided
}

\section{DAPSK/Star-QAM}

\author{
Dandan Liang, Student Member, IEEE, Soon Xin Ng, Senior Member, IEEE, and Lajos Hanzo, Fellow, IEEE \\ Communications Research Group, University of Southampton, SO17 1BJ, U.K. \\ E-Mail: \{dl4e08,sxn,lh\}@ecs.soton.ac.uk
}

\begin{abstract}
Low-complexity non-coherently detected Differential Amplitude and Phase-Shift Keying (DAPSK) schemes constitute an ideal candidate for wireless communications. In this paper, we derive the soft-output probability formulas required for the soft-decision based demodulation of DAPSK, which are then invoked for Turbo Coded (TC) transmissions. Furthermore, the achievable throughput characteristics of the family of M-ary DAPSK schemes are provided. It is shown that the proposed 4ring based TC assisted 64-ary DAPSK scheme achieves a coding gain of about $4.2 \mathrm{dBs}$ in comparison to the identical-throughput TC assisted 64-ary Differential Phase-Shift Keying (64-DPSK) scheme at a bit error ratio of $10^{-5}$.
\end{abstract}

Index Terms-Soft-decision, Iterative detection, DAPSK, TC, Correlated Rayleigh fading channel, Near-capacity transceivers.

\section{INTRODUCTION}

Coherent detection aided square-constellation based Quadrature Amplitude Modulation (QAM) requires accurate Channel State Information (CSI) in order to avoid false phaselocking of the carrier-recovery scheme, especially when communicating over Rayleigh fading channels [1]-[5]. As a remedy, Differential Amplitude and Phase-Shift Keying (DAPSK) was conceived for dispensing with CSI, albeit naturally at the cost of a performance loss. This scheme was termed as Star-QAM in [6]. Non-coherent schemes are particularly beneficial in cooperative communications, where it is unrealistic to estimate all mobile-to-mobile channels [7], [8]. Let us define the notation M-DAPSK $\left(M_{a}, M_{p}\right)$ representing $M_{a}$ amplitudes and $M_{p}$ different phases, which may also be denoted as $M_{a}$-DASK $/ M_{p}$-DPSK or Star-QAM. The authors of [9], [10] have further improved the performance of MDAPSK $\left(M_{a}, M_{p}\right)$ schemes. However, despite its attractive performance versus complexity characteristics, surprisingly soft-decision based demodulation has not been conceived for these M-DAPSK $\left(M_{a}, M_{p}\right)$ schemes. This also implies that without soft-decision based demodulation, the full potential of sophisticated channel coding or coded modulation schemes cannot be entirely exploited. Hence, when channel coding is incorporated into M-DAPSK $\left(M_{a}, M_{p}\right)$ as in [6], its performance is far from the detection-dependent Discrete-input Continuous-output Memoryless Channel (DCMC) capacity due to the employment of hard-decision based demodulation.

The research leading to these results has received funding from the European Union's Seventh Framework Programme ([FP7/20072013]) under grant agreement no [214625]. The financial support of the EPSRC UK in the framework of the IU-ATC and the China-UK project in $4 \mathrm{G}$ wireless communications is also gratefully acknowledged.
The twin-ring based soft-decision assisted Star-QAM scheme was proposed for Iteratively Detected Bit-Interleaved Coded Modulation (BICM-ID) in [11]. As a further improvement, in this contribution Turbo Coding (TC) [12] is employed because its Extrinsic Information Transfer (EXIT) curve shape was found to have a better match with that of the M-DAPSK $\left(M_{a}, M_{p}\right)$ demapper. Which is beneficial, because the smaller the area between these two EXIT curves, the closer this scheme operates to the achievable capacity [8], [14]. Our novel contributions are:

- we will first solve the open problem of deriving the soft-decision demodulation probability formulas for MDAPSK $\left(M_{a}, M_{p}\right)$ schemes, which have more than two concentric PSK constellations;

- Secondly, the performance benefits of using the new formulas will be quantified in the context of TC schemes invoked for communications over correlated Rayleigh fading channels at a normalised Doppler frequency of 0.01 ;

- Finally, the channel capacity of M-DAPSK $\left(M_{a}, M_{p}\right)$ will be quantified for the the sake of demonstrating that our proposed scheme constitutes a near-capacity design.

This paper is organised as follows. In Section II, the softdecision demodulation of M-DAPSK $\left(M_{a}, M_{p}\right)$ aided TC will be presented. Our results will be discussed in Section III and our conclusions are offered in Section IV.

\section{SySTEM Model AND ANALYSis}

Fig. 1 shows the simplified schematic of the near-capacity TC aided M-DAPSK $\left(M_{a}, M_{p}\right)$ scheme, where the number of constellation points is $\mathrm{M}=M_{a} * M_{p}=2^{\mathrm{m}}$, while the number of amplitudes is $M_{a}=2^{m_{a}}$ and the number of phases per amplitude circle is $M_{p}=2^{m_{p}}$. More explicitly, $\mathrm{m}, m_{a}$ and $m_{p}$ denotes the total number of modulated bits/symbol, the number of bits assigned to the amplitude rings and that assigned select the phases, respectively. A sequence of information symbols is encoded by a rate- $1 / 2$ TC encoder for generating a sequence of coded symbols. Out of the total of $\mathrm{m}$ bits, $m_{a}$ bits will be used for selecting the amplitude of the Phase-Shift-Keying (PSK) ring, while the remaining $\left(\mathrm{m}-m_{a}\right)$ bits will be used for selecting the phase of the complex-valued M-DAPSK $\left(M_{a}, M_{p}\right)$ symbol $x_{k}$, where the subscript $k$ denotes the symbol index. As shown in Fig. 1, the TC-encoded M-DAPSK symbol is corrupted by both the complex-valued Rayleigh fading channel coefficient 


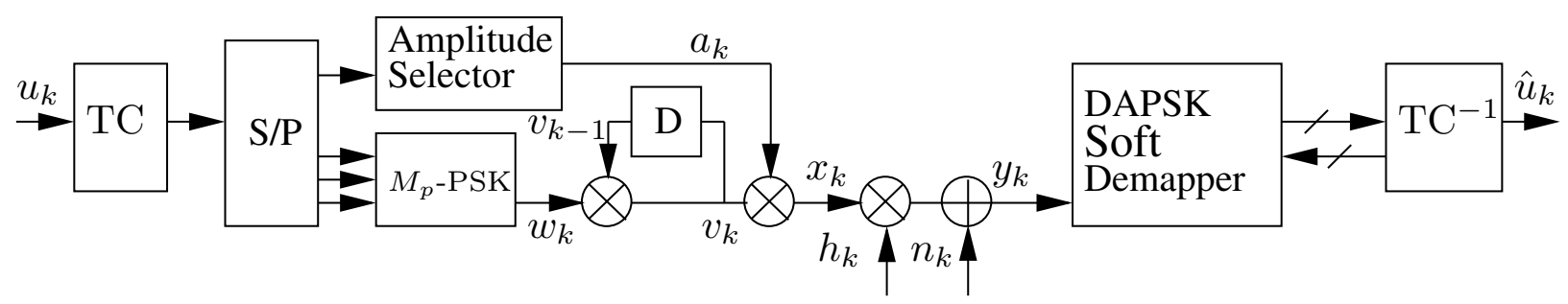

Fig. 1: The schematic of the M-DAPSK $\left(M_{a}, M_{p}\right)$ aided TC scheme, where the interleavers and de-interleavers between the encoder/decoder and mapper/de-mapper are not shown for simplicity.

$h_{k}$ and the Additive White Gaussian Noise (AWGN) $n_{k}$, when it is transmitted to the receiver. Iterative detection is then carried out by exchanging extrinsic information between the M-DAPSK soft demapper and TC decoder based on the received sequence $\left\{y_{k}\right\}$ without exploiting any CSI.

\section{A. M-DAPSK Mapper}

As seen in Fig. 1, the M-DAPSK $\left(M_{a}, M_{p}\right)$ mapper consists of three components, namely the amplitude selector, the $M_{p^{-}}$ PSK mapper and a differential encoder. The $M_{p}$-PSK mapper and the differential encoder jointly form a conventional $M_{p}$-level DPSK ( $M_{p}$-DPSK) mapper. The $m_{a}$ bits of the TC-encoded symbol are used for selecting one of the $M_{a}$ possible amplitude circles. The remaining $\left(\mathrm{m}-m_{a}\right)$ bits are used by the $M_{p}$-DPSK mapper. Note that similar to any DPSK scheme, we insert a reference symbol at the beginning of each differentially encoded transmission frame before the M-DAPSK $\left(M_{a}, M_{p}\right)$ mapper. We use the 64-DAPSK $(4,16)$ scheme as an example for illustrating the philosophy of our proposed soft-decision based demapper.

1) Amplitude Selection: $m_{a}=2$ bits are used for selecting the amplitude of the PSK ring, $a_{k}$. The four possible amplitude values are denoted as $a^{(1)}, a^{(2)}, a^{(3)}$ and $a^{(4)}$, respectively. Table I illustrates the procedure of amplitude selection. Note that the classic Set Partitioning (SP) [13] method is employed in the mapper. This amplitude selection mechanism may be

\begin{tabular}{|c|c|c|c|c|c|}
\hline \multirow{2}{*}{\multicolumn{2}{|c|}{$a_{k}$}} & \multicolumn{4}{|c|}{$m_{a}$-bits $\left(b_{5}\right.$ and $\left.b_{4}\right)$} \\
\hline & & 00 & 01 & 10 & 11 \\
\hline \multirow{4}{*}{$a_{k-1}$} & $a^{(1)}$ & $a^{(1)}$ & $a^{(2)}$ & $a^{(3)}$ & $a^{(4)}$ \\
\hline & $a^{(2)}$ & $a^{(2)}$ & $a^{(3)}$ & $a^{(4)}$ & $a^{(1)}$ \\
\hline & $a^{(3)}$ & $a^{(3)}$ & $a^{(4)}$ & $a^{(1)}$ & $a^{(2)}$ \\
\hline & $a^{(4)}$ & $a^{(4)}$ & $a^{(1)}$ & $a^{(2)}$ & $a^{(3)}$ \\
\hline
\end{tabular}

TABLE I: Table to generate the amplitude $a_{k}$.

referred to as $M_{a}$-level Differential Amplitude Shift Keying ( $M_{a}$-DASK). After normalisation to a symbol energy of unity, we have $a^{(1)}=1 / \sqrt{3.58}, a^{(2)}=1.4 / \sqrt{3.58}, a^{(3)}=$ $(1.4)^{2} / \sqrt{3.58}$ and $a^{(4)}=(1.4)^{3} / \sqrt{3.58}$. The amplitude value of the reference symbol is given by $a_{0}=a^{(1)}$. Fig. 2 shows the constellation diagrams of 8-DAPSK $(4,2)$, 16-DAPSK $(4,4), 32$-DAPSK $(4,8)$ and 64-DAPSK $(4,16)$.

2) Phase Selection: When we consider $m_{p}=4$, the $k$ th differentially encoded symbol $v_{k}$ can be expressed as:

$$
v_{k}=v_{k-1} w_{k},
$$
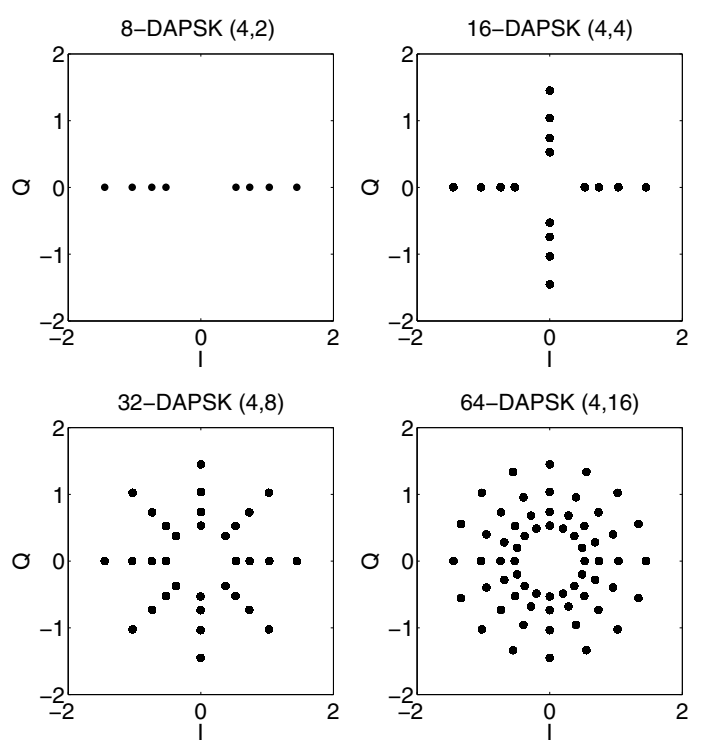

Fig. 2: Constellation diagrams of the M-DAPSK $\left(4, M_{p}\right)$ modulation schemes.

where $x_{k}=\mu\left(\begin{array}{llll}b_{3} & b_{2} & b_{1} & b_{0}\end{array}\right)$ is the $k$ th 16-PSK symbol based on the 16-PSK mapping function of $\mu($.$) , while v_{k-1}$ is the $(k-1)$ st 16-DPSK symbol and $\left|v_{k}\right|^{2}=1$. The reference symbol of the 16-DPSK part of the constellation is given by $v_{0}=\mu\left(\begin{array}{llll}0 & 0 & 0 & 0\end{array}\right)$.

The $k$ th $64-D A P S K$ symbol is then given by:

$$
x_{k}=a_{k} v_{k},
$$

where we have $a_{k} \in\left\{a^{(1)}, a^{(2)}, a^{(3)}, a^{(4)}\right\}$.

\section{B. M-DAPSK Soft Demapper}

As in all transceivers, the soft-decision based M-DAPSK $\left(M_{a}, M_{p}\right)$ block is placed before the TC decoder, as shown in Fig. 1. The $k$ th received symbol may then be written as:

$$
y_{k}=h_{k} x_{k}+n_{k}=h_{k} a_{k} v_{k}+n_{k},
$$

where $h_{k}$ is the Rayleigh fading channel's coefficient, while $n_{k}$ represents the AWGN having a variance of $N_{0} / 2$ per dimension. Assuming a slowly Rayleigh fading channel, 
where we have $h_{k} \approx h_{k-1}$, we can rewrite (3) using (1) as:

$$
\begin{aligned}
y_{k} & =h_{k-1} a_{k} v_{k-1} w_{k}+n_{k}, \\
& =\frac{a_{k}}{a_{k-1}}\left(y_{k-1}-n_{k-1}\right) w_{k}+n_{k}, \\
& =p_{k} y_{k-1} w_{k}+\tilde{n}_{k}
\end{aligned}
$$

where $p_{k}=\frac{a_{k}}{a_{k-1}}$ is the ratio of the $k$ th and $(k-1)$ st amplitudes, while $\tilde{n}_{k}=-\frac{a_{k}}{a_{k-1}} n_{k-1} w_{k}+n_{k}$ is the effective noise.

1) Amplitude Detection: $\left(2 \cdot M_{a}-1\right)$ amplitude ratios can be derived from the $M_{a}$-PSK ring amplitudes of M-DAPSK $\left(M_{a}, M_{p}\right)$. It may be readily shown that for 64 -DAPSK $(4,16)$, we have:

$$
p_{k}= \begin{cases}R_{(-3)} & =a^{(1)} / a^{(4)} \\ R_{(-2)} & =a^{(1)} / a^{(3)}=a^{(2)} / a^{(4)} \\ R_{(-1)} & =a^{(1)} / a^{(2)}=a^{(2)} / a^{(3)}=a^{(3)} / a^{(4)} \\ R_{(0)} & =a^{(1)} / a^{(1)}=a^{(2)} / a^{(2)} \\ & =a^{(3)} / a^{(3)}=a^{(4)} / a^{(4)} \\ R_{(1)} & =a^{(2)} / a^{(1)}=a^{(3)} / a^{(2)}=a^{(4)} / a^{(3)} \\ R_{(2)} & =a^{(3)} / a^{(1)}=a^{(4)} / a^{(2)} \\ R_{(3)} & =a^{(4)} / a^{(1)} .\end{cases}
$$

When the noise power is low, the amplitude ratio $p_{k}$ may be approximated as:

$$
\begin{aligned}
\frac{\left|y_{k}\right|}{\left|y_{k-1}\right|} & =\frac{\left|h_{k} a_{k} v_{k}+n_{k}\right|}{\left|h_{k-1} a_{k-1} v_{k-1}+n_{k-1}\right|}, \\
& \approx \frac{\left|a_{k}\right|}{\left|a_{k-1}\right|} \\
& \approx p_{k} .
\end{aligned}
$$

2) Probability Computation: The effective noise variance of $\tilde{n}_{k}$ in (4) depends on the amplitude ratio used at time instant $k$, which can be computed as:

$$
\tilde{N}_{0}=N_{0}+\left|p_{k}\right|^{2}\left|w_{k}\right|^{2} N_{0}=N_{0}\left(1+\left|p_{k}\right|^{2}\right),
$$

where $\quad \tilde{N}_{0}=\left(1+R_{(q)}^{2}\right) N_{0}=N_{0}^{(q)}, \quad \mathrm{q} \quad \in$ $\{-3,-2,-1,0,1,2,1,3\}$. Based on (4) we can express the probability of receiving $y_{k}$ conditioned on the transmission of $b_{0}, b_{1}, b_{2}, b_{3}, b_{4}$ and $b_{5}$ as in (9). The bit-probabilities then can be converted to the Log-Likelihood Ratio (LLR) [14] based representations of $b_{i}, i \in\{0,1,2,3,4,5\}$ where we have $w^{(m)}=\mu\left(b_{3} b_{2} b_{1} b_{0}\right)$ and $\mu$ is the conventional 16-PSK mapper function.

$$
\begin{aligned}
P\left(y_{k} \mid w^{(m)}, b_{5} b_{4}=00\right) & =\frac{1}{\pi N_{0}^{(0)}} e^{\frac{-\left|y_{k}-y_{k-1} R_{(0)}(m)\right|^{2}}{N_{0}^{(0)}}}, \\
P\left(y_{k} \mid w^{(m)}, b_{5} b_{4}=01\right) & =\frac{1}{\pi N_{0}^{(1)}} e^{\frac{-\left|y_{k}-y_{k-1} R_{(1)} w^{(m)}\right|^{2}}{N_{0}^{(1)}}} \\
& +\frac{1}{\pi N_{0}^{(-3)}} e^{\frac{-\left|y_{k}-y_{k-1} R_{(-3)} w^{(m)}\right|^{2}}{N_{0}^{(-3)}}}, \\
P\left(y_{k} \mid w^{(m)}, b_{5} b_{4}=10\right) & =\frac{1}{\pi N_{0}^{(2)}} e^{\frac{-\left|y_{k}-y_{k-1} R_{(2)}(m)\right|^{2}}{N_{0}^{(2)}}} \\
& +\frac{1}{\pi N_{0}^{(-2)}} e^{\frac{-\left|y_{k}-y_{k-1} R_{(-2)} w^{(m)}\right|^{2}}{N_{0}^{(-2)}}}, \\
P\left(y_{k} \mid w^{(m)}, b_{5} b_{4}=11\right) & \frac{1}{\pi N_{0}^{(-1)}} e^{\frac{-\left|y_{k}-y_{k-1} R_{(3)}(m)\right|^{2}}{N_{0}^{(3)}}} \\
& +
\end{aligned}
$$

\begin{tabular}{|c|c|}
\hline Modulation & $\begin{array}{r}\text { 64-DAPSK }(4,16), \text { 32-DAPSK }(4,8), \text { 16-DAPSK }(4,4) \\
\text { 8-DAPSK }(4,2), 64-\mathrm{QAM}, 64-\mathrm{DPSK}\end{array}$ \\
\hline Mapping & Set Partitioning (SP) \\
\hline Coding & TC \\
\hline $\begin{array}{l}\text { Constituent } \\
\text { Code }\end{array}$ & $\begin{array}{r}\text { Half-rate Recursive Systematic Convolutional (RSC) code } \\
\text { Code Polynomial G=[15 17] }\end{array}$ \\
\hline $\begin{array}{l}\text { Code } \\
\text { Memory }\end{array}$ & 3 \\
\hline $\begin{array}{l}\text { Outer iter- } \\
\text { ations }\end{array}$ & 2 \\
\hline $\begin{array}{l}\text { Inner } \mathrm{TC} \\
\text { iterations }\end{array}$ & 4 \\
\hline Decoder & Approximate Log-MAP \\
\hline $\begin{array}{l}\text { Symbols } \\
\text { per 64- } \\
\text { DAPSK } \\
\text { block }\end{array}$ & 400,4000 \\
\hline $\begin{array}{l}\text { Number } \\
\text { of 64- } \\
\text { DAPSK } \\
\text { blocks per } \\
\text { TC block }\end{array}$ & 1,10 \\
\hline $\begin{array}{l}\text { Number of } \\
\text { TC blocks }\end{array}$ & 5000 \\
\hline Channel & $\begin{array}{l}\text { Correlated Rayleigh fading channel } \\
\text { having a normalised Doppler frequency of } 0.01\end{array}$ \\
\hline
\end{tabular}

\section{Simulation Results}

Computer simulations have been performed for characterising the proposed soft-decision based M-DAPSK $\left(4, M_{p}\right)$ demodulation technique in the context of TC coding schemes. In order to benchmark our proposed method, the classic square-constellation 64-QAM and 64-DPSK schemes were employed. The simulation parameters are shown in Table II.

TABLE II: System parameters.

Firstly, the EXIT Charts of the proposed system is presented for analysing the 64-DAPSK $(4,16)$ scheme. Fig. 3 illustrates 


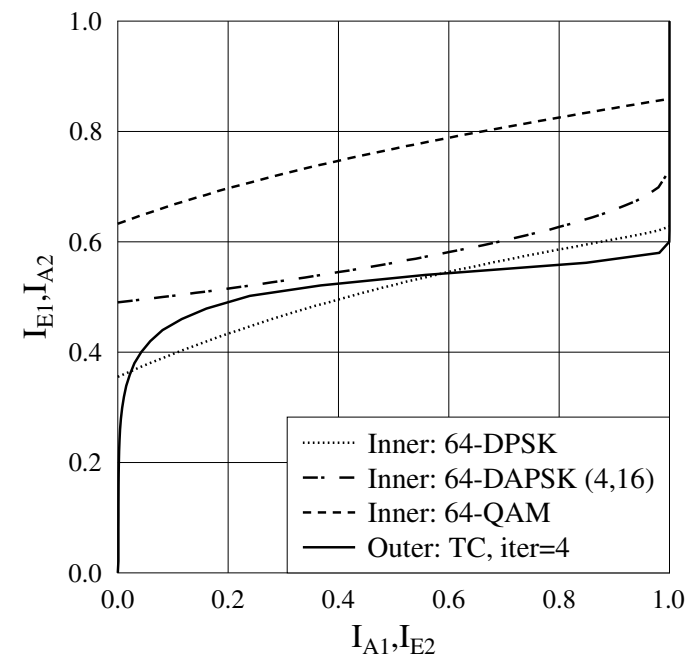

Fig. 3: EXIT Charts of the 64-QAM, 64-DAPSK $(4,16)$ and 64-DPSK aided TC schemes when communicating over a correlated Rayleigh channel at $\mathrm{SNR}=17.5 \mathrm{~dB}$. The SNRindependent EXIT curve of the outer TC decoder is also shown. The corresponding simulation parameters are presented in Table. II.

three inner decoder ${ }^{1}$ EXIT curves, namely those of the 64QAM, 64-DAPSK $(4,16)$ and 64-DPSK schemes, together with the EXIT curve of the outer TC decoder. It is worth noting that the area under the EXIT curve of the inner decoder is approximately equal to the channel capacity [7], [15], [16]. It is then clear from Fig. 3 that the area under the square-constellation 64-QAM scheme's EXIT curve is larger than that under the 64-DAPSK $(4,16)$ scheme's curve, which is in turn higher than that of the 64-DPSK arrangement. It was also demonstrated in [7], [8] that an open EXIT chart tunnel implies having an infinitesimally low BER. Hence we may argue based on Fig. 3 that a vanishingly low BER may be achieved by the TC aided 64-DAPSK $(4,16)$ scheme for SNR values in excess of $17.5 \mathrm{~dB}$. By contrast, no open EXIT chart tunnel is maintained for the same SNR value in the case of the 64-DPSK benchmark scheme.

The corresponding BER versus SNR performance is depicted in Fig. 4, which compares the performance of the 64-QAM, 64-DAPSK $(4,16)$ and 64-DPSK aided TC schemes, when communicating over correlated Rayleigh fading channels using different transmission block lengths and turbointerleaved block lengths. More explicitly, as seen in Table II, each block consists of 400 or 4000 modulated symbols. The TC block length is given by the number of modulated symbols per 64-DAPSK transmission block times the number of transmission blocks per TC block. When the number of 64DAPSK modulated transmission blocks per TC block is one, which corresponds to the curve marked by circles in Fig. 4, the SNR difference between the classic coherently detected

${ }^{1}$ In serially concatenated and turbo-detected schemes the softoutput demodulator is often referred to as the inner decoder, for the sake of a unified terminology.

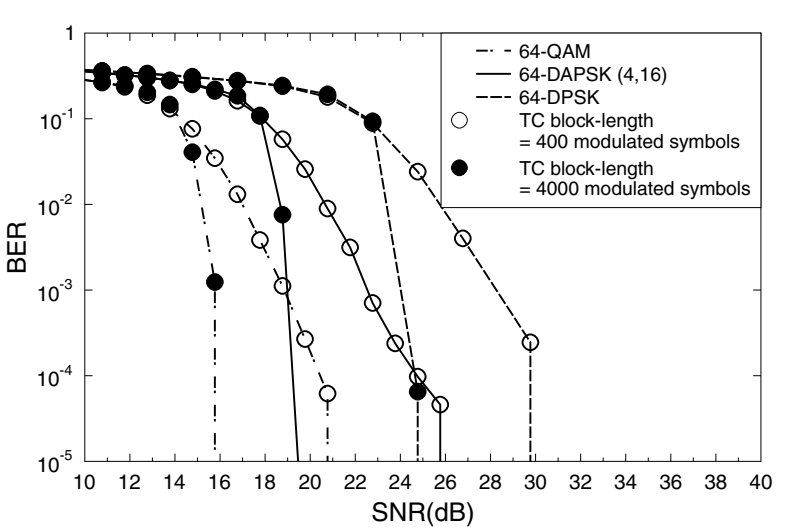

Fig. 4: BER versus SNR (dB) performance comparison of the 64-QAM, 64-DAPSK $(4,16)$ and 64-QAM schemes for transmission over correlated Rayleigh fading channels. The corresponding system parameters are summarized in Table. II. A TC block-length of 400 modulated symbols corresponds to one 64-DAPSK block-length, while a 4000-modulated-symbol TC block corresponds to ten 64-DAPSK block-length.

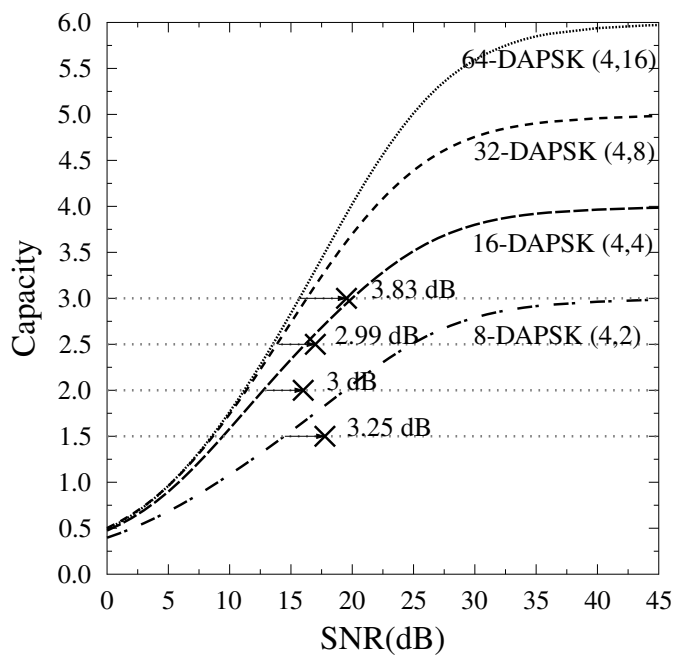

Fig. 5: Achievable throughput versus SNR $(\mathrm{dB})$ for with the systems of Fig. 2 for transmission over correlated Rayleigh fading channels, while using TC block length of 4000 modulated symbols.

square-constellation 64-QAM and our low-complexity 64DAPSK dispensing with power-thirsty channel-estimation is 5 dB. As a substantial further benefit, our scheme outperforms 64-DPSK by about $4.2 \mathrm{~dB}$. When the number of 64-DAPSK blocks per TC block is increased to ten (the curve marked by filled circles), all the BER performances are improved, but the SNR differences remain similar. In general, the longer the TC block-length, the closer the BER performance to the channel capacity.

Finally, the achievable throughput of the M-DAPSK (4, $M_{p}$ ) modem family is characterised in Fig. 5, where the curves were generated by evaluating the area under the 
corresponding EXIT curves, as detailed in [8], [14]. The large crosses represent the SNR required for the corresponding modulation schemes to achieve an identical throughput to each other at a target BER of $10^{-5}$. The horizontal grey dotted lines represent the throughput values of the different modulation schemes considered. For example, 1.5 is that of TC aided 8-DAPSK $(4,2)$. Fig. 5 also shows the SNR discrepancy between the various schemes and the corresponding channel capacity. More specifically, the TC aided 64-DAPSK $(4,16)$ scheme is capable of reliably operating approximately 3.83 $\mathrm{dB}$ away from its capacity, while the TC aided 32-DAPSK $(4,8)$ arrangement operates approximately $2.99 \mathrm{~dB}$ away from its capacity.

\section{CONCLUSIONS}

The symbol-to-bit soft-demapper probability formulas of the 64-DAPSK $(4,16)$ scheme were derived and its softdecision aided performance was investigated in the context of a TC scheme. The 64-DAPSK-TC scheme outperforms the identical-throughput 64-DPSK-TC scheme by about $4.2 \mathrm{~dB}$ at a BER of $10^{-5}$, when communicating over correlated Rayleigh fading channels having a normalised Doppler frequency of 0.01. Finally, the achievable throughput characteristics of the M-DAPSK $\left(4, M_{p}\right)$ modem-family were presented. Our future research will improve the resilience of these schemes against high Doppler frequencies with the aid of multiplesymbol differential detection (MSDD) [8]. We will also conceive low-complexity MSDD schemes with the aid of sphere decoding [8].

\section{REFERENCES}

[1] E. Issman and W. Webb, "Carrier recovery for 16-level QAM in mobile radio," IEE colloquium on multi-level modulation, pp. $9 / 1-9 / 8$, March 1990.

[2] L. Hanzo, S. X. Ng, T. Keller, and W. Webb, Quadrature Amplitude Modulation: From Basics to Adaptive Trellis-Coded, Turbo-Equalised and Space-Time Coded OFDM, CDMA and MC-CDMA Systems Digital Communications, 2nd ed. WileyIEEE Press, 2004.

[3] L. Chen, H. Kusaka, and M. Kominami, "Blind phase recovery in QAM communication systems using higher order statistics," Signal Processing Letters, IEEE, vol. 3, no. 5, pp. $147-149$, may. 1996.

[4] Y. Wang and E. Serpedin, "A class of blind phase recovery techniques for higher order QAM modulations: estimators and bounds," Signal Processing Letters, IEEE, vol. 9, no. 10, pp. $301-304$, oct. 2002.

[5] H. Rohling and V. Engels, "Differential amplitude phase shift keying (dapsk)-a new modulation method for dtvb," in Broadcasting Convention, 1995. IBC 95., International, Sept. 1995, pp. $102-108$.

[6] W. Webb, L. Hanzo, and R. Steele, "Bandwidth-efficient QAM schemes for Rayleigh-fading channels," IEE Proceedings, vol. 138, no. 3, pp. 169-175, June 1991.

[7] L. Hanzo and O. Alamri and N. El-Hajjar and N. Wu, NearCapacity Multi Functional MIMO Systems. John Wiley \& Sons, Ltd, May 2009.

[8] L. Hanzo, Y. Akhtman, L. Wang, M. Jiang, MIMO-OFDM for LTE, WiFi and WiMAX: Coherent versus Non-coherent and Cooperative Turbo Transceivers. John Wiley \& Sons, Ltd, October 2010
[9] C.-D. Chung, "Differentially amplitude and phase-encoded QAM for the correlated Rayleigh-fading channel with diversity reception," IEEE Transactions on Communications, vol. 45, no. 3, pp. 309 - 321, March 1997.

[10] Y. Ma, Q. T. Zhang, R. Schober and S. Pasupathy, "Diversity reception of DAPSK over generalized fading channels," IEEE Transactions on Wireless Communications, vol. 4, no. 4, pp. 1834 - 1846, July 2005.

[11] D. Liang, S. X. Ng, and L. Hanzo, "Soft-decision Star-QAM aided BICM-ID," Signal Processing Letters, IEEE, vol. 18, no. 3, pp. $169-172,2011$.

[12] C. Berrou and A. Glavieux and P. Thitimajshima, "Near Shannon limit error-correcting coding and decoding: Turbo codes," in Proceedings of the International Conference on Communications, Geneva, Switzerland, May 1993, pp. 1064 1070.

[13] M. Rohling, T. May, K. Bruninghaus, and R. Grunheid, "Broadband OFDM radio transmission for multimedia applications," Proceedings of the IEEE, vol. 87, no. 10, pp. 1778 -1789, Oct. 1999.

[14] L. Hanzo, T. H. Liew, B. L. Yeap, R. Y. S. Tee, S. $\mathrm{X}$. Ng, Turbo Coding, Turbo Equalisation and Space-Time Coding: EXIT-Chart-Aided Near-Capacity Designs for Wireless Channels, 2nd Edition. Wiley-IEEE Press, 2011.

[15] S. ten Brink, "Convergence behaviour of iteratively decoded parallel concatenated codes," IEEE Transactions on Communications, vol. 49, no. 10, pp. 1727-1737, October 2001.

[16] J. Kliewer, S. X. Ng, and L. Hanzo, "Efficient computation of EXIT functions for non-binary iterative decoding," IEEE Transactions on Communications, vol. 54, no. 12, pp. $2133-$ 2136, December 2006. 